\title{
Production of massive particles during reheating
}

\author{
Daniel J. H. Chung* \\ NASA/Fermilab Astrophysics Center Fermilab National Accelerator Laboratory, Batavia, Illinois 60510-0500 \\ and Department of Physics, Enrico Fermi Institute, The University of Chicago, Chicago, Illinois 60637-1433 \\ Edward W. Kolb \\ NASA/Fermilab Astrophysics Center Fermilab National Accelerator Laboratory, Batavia, Illinois 60510-0500 \\ and Department of Astronomy and Astrophysics, Enrico Fermi Institute, The University of Chicago, Chicago, Illinois 60637-1433
}

Antonio Riotto

Theory Division, CERN, CH-1211 Geneva 23, Switzerland

(Received 23 September 1998; published 12 August 1999)

\begin{abstract}
What is commonly called the reheat temperature, $T_{R H}$, is not the maximum temperature obtained after inflation. The maximum temperature is, in fact, much larger than $T_{R H}$. As an application of this we consider the production of massive stable dark-matter particles of mass $M_{X}$ during reheating, and show that their abundance is suppressed as a power of $T_{R H} / M_{X}$ rather than $\exp \left(-M_{X} / T_{R H}\right)$. We find that particles of mass as large as $2 \times 10^{3}$ times the reheat temperature may be produced in interesting abundance. In addition to dark matter, our analysis is relevant for baryogenesis if the baryon asymmetry is produced by the baryon (or lepton) number violating decays of superheavy bosons, and also for relic ultra-high energy cosmic rays if decays of superheavy particles are responsible for the highest energy cosmic rays. [S0556-2821(99)07918-7]
\end{abstract}

PACS number(s): $98.80 . \mathrm{Cq}$

\section{INTRODUCTION}

At the end of inflation [1] the energy density of the universe is locked up in a combination of kinetic energy and potential energy of the inflaton field, with almost all of the inflaton energy density in the zero-momentum mode of the field. Thus, the universe at the end of inflation is in a cold, low-entropy state with few degrees of freedom, very much unlike the present hot, high-entropy universe. After inflation the frozen inflaton-dominated universe must somehow defrost and become a high-entropy radiation-dominated universe. It is now appreciated that defrosting may occur through a combination of linear and nonlinear processes. We will refer to nonlinear effects in defrosting as "preheating" [2] and refer to linear processes as "reheating" [3].

The possible role of nonlinear dynamics leading to explosive particle production has recently received a lot of attention. This process, known as "preheating" [2] may convert a fair fraction of the inflaton energy density into other degrees of freedom, with extremely interesting cosmological effects such as symmetry restoration, baryogenesis, or production of dark matter. But the efficiency of preheating is very sensitive to the model and the model parameters.

The parameters of the model may be such that the parametric resonance and the preheating period does not occur at all. For instance, if the inflaton field is very weakly coupled, say with a decay rate suppressed by powers of the Planck

\footnotetext{
*Electronic mail: djchung@ theory.uchicago.edu

${ }^{\dagger}$ Electronic mail: rocky@rigoletto.fnal.gov

* On leave from Department of Theoretical Physics, University of Oxford, U.K. Electronic mail: riotto@nxth04.cern.ch
}

scale, preheating will not occur. ${ }^{1}$

It may also be the case that the parameters of the model are such that there is a short period of preheating that does not fully extract all of the energy density from the inflaton field. Even if there is a short period of preheating, the universe is likely to enter a long period of matter domination where the dominant contribution to the energy density of the universe is provided by the residual small amplitude oscillations of the classical inflaton field and/or by the inflaton quanta produced during the back-reaction processes. This period will end when the age of the universe becomes of the order of the perturbative lifetime of the inflaton field. At this point the universe will go through a period of reheating with a reheat temperature $T_{R H}$ obtained by applying the perturbative theory of reheating.

Therefore, one may consider two different possibilities. Either there is a short period of preheating, which is inevitably followed by a long period of matter-domination and by the release of energy under the form of radiation in the usual perturbative way, or preheating does not take place, and again the perturbative analysis of reheating applies. In either case, it should be clear that our paper deals with the reheating period in which the perturbative analysis holds.

The simplest way to envision the process of reheating is if the comoving energy density in the zero mode of the inflaton decays into normal particles, which then scatter and thermalize to form a thermal background. It is usually assumed that the decay width of this process is the same as the decay width of a free inflaton field.

\footnotetext{
${ }^{1}$ Again, we emphasize that we will use the term "preheating" to refer to nonlinear effects involved in defrosting, and reserve the term "reheating'" to describe linear processes.
} 
There are two reasons to suspect that the inflaton decay width might be small. The requisite flatness of the inflaton potential suggests a weak coupling of the inflaton field to other fields since the potential is renormalized by the inflaton coupling to other fields [1]. However, this restriction may be evaded in supersymmetric theories where the nonrenormalization theorem ensures a cancellation between fields and their superpartners. A second reason to suspect weak coupling is that in local supersymmetric theories gravitinos are produced during reheating. Unless reheating is delayed, gravitinos will be over produced, leading to a large undesired entropy production when they decay after big-bang nucleosynthesis [4].

Of particular interest is a quantity known as the reheat temperature, denoted as $T_{R H}$. The reheat temperature is calculated by assuming an instantaneous conversion of the energy density in the inflaton field into radiation when the decay width of the inflaton energy, $\Gamma_{\phi}$, is equal to $H$, the expansion rate of the universe.

The reheat temperature is calculated quite easily [3]. After inflation the inflaton field executes coherent oscillations about the minimum of the potential. Averaged over several oscillations, the coherent oscillation energy density redshifts as matter: $\rho_{\phi} \propto a^{-3}$, where $a$ is the Robertson-Walker scale factor. If we denote as $\rho_{I}$ and $a_{I}$ the total inflaton energy density and the scale factor at the initiation of coherent oscillations, then the Hubble expansion rate as a function of $a$ is ( $M_{P l}$ is the Planck mass)

$$
H(a)=\sqrt{\frac{8 \pi}{3} \frac{\rho_{I}}{M_{P l}^{2}}\left(\frac{a_{I}}{a}\right)^{3}} .
$$

Equating $H(a)$ and $\Gamma_{\phi}$ leads to an expression for $a_{I} / a$. Now if we assume that all available coherent energy density is instantaneously converted into radiation at this value of $a_{I} / a$, we can define the reheat temperature by setting the coherent energy density, $\rho_{\phi}=\rho_{I}\left(a_{I} / a\right)^{3}$, equal to the radiation energy density, $\rho_{R}=\left(\pi^{2} / 30\right) g_{*} T_{R H}^{4}$, where $g_{*}$ is the effective number of relativistic degrees of freedom at temperature $T_{R H}$. The result is

$$
T_{R H}=\left(\frac{90}{8 \pi^{3} g_{*}}\right)^{1 / 4} \sqrt{\Gamma_{\phi} M_{P l}}=0.2\left(\frac{200}{g_{*}}\right)^{1 / 4} \sqrt{\Gamma_{\phi} M_{P l}} .
$$

The limit from gravitino overproduction is $T_{R H} \lesssim 10^{9}$ to $10^{10}$ $\mathrm{GeV}$.

The reheat temperature is best regarded as the temperature below which the universe expands as a radiation-dominated universe, with the scale factor decreasing as $g_{*}^{-1 / 3} T^{-1}$. In this regard it has a limited meaning [3,5]. For instance, $T_{R H}$ should not be used as the maximum temperature obtained by the universe during reheating. The maximum temperature is, in fact, much larger than $T_{R H}$. One implication of this is that it is incorrect to assume that the maximum abundance of a massive particle species produced after inflation is suppressed by a factor of $\exp \left(-M / T_{R H}\right)$.

In this paper we illustrate this effect by calculating the abundance of a massive particle species produced in reheat- ing. We show that particles of mass much greater than the eventual "reheat" temperature $T_{R H}$ may be created by the thermalized decay products of the inflaton. As an example, we demonstrate that a stable particle species $X$ of mass $M_{X}$ would be produced in the reheating process in sufficient abundance that its contribution to closure density today is approximately $M_{X}^{2}\langle\sigma|\mathrm{v}|\rangle\left(g_{*} / 200\right)^{-3 / 2}\left(2000 T_{R H} / M_{X}\right)^{7}$, where $g_{*}$ is the number of effective degrees of freedom of the radiation energy density and $\langle\sigma|\mathrm{v}|\rangle$ is the thermal average of the $X$ annihilation cross section times the Møller flux factor. Thus, particles of mass as large as 2000 times the reheat temperature may be produced in interesting abundance.

Other applications of the effect include production of massive Higgs bosons which could decay and produce the baryon asymmetry, or massive particles that could decay and produce high-energy cosmic rays.

In the next section we develop a system of Boltzmann equations describing the evolution of the energy densities of the inflaton field, radiation, and a massive particle species. In Sec. III we find analytic approximations to the system and estimate the contribution to the present critical density from a stable, massive particle produced in reheating. Section IV contains some numerical results which illustrate several generic features. We conclude in Sec. V by discussing some applications of our results. The assumption of local thermodynamic equilibrium for the light degrees of freedom is addressed in an appendix.

\section{BOLTZMANN EQUATIONS DESCRIBING REHEATING}

Let us consider a model universe with three components: inflaton field energy, $\rho_{\phi}$, radiation energy density, $\rho_{R}$, and the energy density of a nonrelativistic particle species, $\rho_{X}$. We will assume that the decay rate of the inflaton field energy density is $\Gamma_{\phi}$, with a branching fraction into $X \bar{X}$ of $B_{X}$, and a branching fraction $1-B_{X}$ into light degrees of freedom, generically referred to as radiation. We will denote the decay width of the $X$ as $\Gamma_{X}$. We will also assume that the light degrees of freedom are in local thermodynamic equilibrium. This is by no means guaranteed, and we will return to the question in the Appendix. A final assumption is that the equilibrium distribution function of the heavy $X$-particles is well approximated by the non-relativisitic one throughout the reheating process. As we shall see, this approximation is well justified.

With the above assumptions, the Boltzmann equations describing the redshift and interchange in the energy density among the different components is

$$
\begin{gathered}
\dot{\rho}_{\phi}+3 H \rho_{\phi}+\Gamma_{\phi} \rho_{\phi}=0 \\
\dot{\rho}_{R}+4 H \rho_{R}-\left(1-B_{X}\right) \Gamma_{\phi} \rho_{\phi}-\frac{\langle\sigma|\mathrm{v}|\rangle}{M_{X}}\left[\rho_{X}^{2}-\left(\rho_{X}^{E Q}\right)^{2}\right] \\
-\Gamma_{X}\left(\rho_{X}-\rho_{X}^{E Q}\right)=0
\end{gathered}
$$




$$
\begin{aligned}
\dot{\rho}_{X}+ & 3 H \rho_{X}-B_{X} \Gamma_{\phi} \rho_{\phi}+\frac{\langle\sigma|\mathrm{v}|\rangle}{M_{X}}\left[\rho_{X}^{2}-\left(\rho_{X}^{E Q}\right)^{2}\right] \\
& +\Gamma_{X}\left(\rho_{X}-\rho_{X}^{E Q}\right)=0
\end{aligned}
$$

where the dot denotes time derivative As already mentioned, $\langle\sigma|\mathrm{v}|\rangle$ is the thermal average of the $X$ annihilation cross section times the M $\phi$ ller flux factor. The equilibrium energy density for the $X$ particles, $\rho_{X}^{E Q}$, is determined by the radiation temperature, $T$.

It is useful to introduce two dimensionless constants, $\alpha_{\phi}$ and $\alpha_{X}$, defined in terms of $\Gamma_{\phi}$ and $\langle\sigma|\mathrm{v}|\rangle$ as

$$
\Gamma_{\phi}=\alpha_{\phi} M_{\phi}, \quad\langle\sigma|\mathrm{v}|\rangle=\alpha_{X} M_{X}^{-2} .
$$

For a reheat temperature much smaller than $M_{\phi}, \Gamma_{\phi}$ must be small. From Eq. (2), the reheat temperature in terms of $\alpha_{X}$ and $M_{X}$ is $T_{R H} \simeq \alpha_{\phi}^{1 / 2} \sqrt{M_{\phi} M_{P l}}$. For $M_{\phi}=10^{13} \mathrm{GeV}, \alpha_{\phi}$ must be smaller than of order $10^{-13}$. With the parametrization of $\langle\sigma|\mathrm{v}|\rangle$ in Eq. (4), unitarity requires $\alpha_{X}$ to be smaller than of order unity. Of course, it may be much smaller than unity.

In what follows we will make the simplifying assumption that $B_{X}=0$. Since we are interested in a stable particle relic, we will assume that $\Gamma_{X}=0$. It is also convenient to work with dimensionless quantities that can absorb the effect of expansion of the universe. This may be accomplished with the definitions

$$
\Phi \equiv \rho_{\phi} M_{\phi}^{-1} a^{3} ; \quad R \equiv \rho_{R} a^{4} ; \quad X \equiv \rho_{X} M_{X}^{-1} a^{3} .
$$

It is also convenient to use the scale factor, rather than time, for the independent variable, so we define a variable $x$ $=a M_{\phi}$. With this choice the system of equations can be written as (prime denotes $d / d x$ )

$$
\begin{aligned}
& \Phi^{\prime}=-c_{1} \frac{x}{\sqrt{\Phi x+R}} \Phi \\
& R^{\prime}=c_{1} \frac{x^{2}}{\sqrt{\Phi x+R}} \Phi+c_{2} \frac{x^{-1}}{\sqrt{\Phi x+R}}\left(X^{2}-X_{E Q}^{2}\right) \\
& X^{\prime}=-c_{3} \frac{x^{-2}}{\sqrt{\Phi x+R}}\left(X^{2}-X_{E Q}^{2}\right) .
\end{aligned}
$$

The constants $c_{1}, c_{2}$, and $c_{3}$ are given by

$$
c_{1}=\sqrt{\frac{3}{8 \pi}} \frac{M_{P l}}{M_{\phi}} \alpha_{\phi}, \quad c_{2}=c_{1} \frac{M_{\phi}}{M_{X}} \frac{\alpha_{X}}{\alpha_{\phi}}, \quad c_{3}=c_{2} \frac{M_{\phi}}{M_{X}} .
$$

$X_{E Q}$ is the equilibrium value of $X$, given in terms of the temperature $T$ as (assuming a single degree of freedom for the $X$ species)

$$
X_{E Q}=\frac{M_{X}^{3}}{M_{\phi}^{3}}\left(\frac{1}{2 \pi}\right)^{3 / 2} x^{3}\left(\frac{T}{M_{X}}\right)^{3 / 2} \exp \left(-M_{X} / T\right) .
$$

The temperature depends upon $R$ and $g_{*}$, the effective number of degrees of freedom in the radiation:

$$
\frac{T}{M_{X}}=\left(\frac{30}{g_{*} \pi^{2}}\right)^{1 / 4} \frac{M_{\phi}}{M_{X}} \frac{R^{1 / 4}}{x} .
$$

It is straightforward to solve the system of equations in Eq. (6) with initial conditions at $x=x_{I}$ of $R\left(x_{I}\right)=X\left(x_{I}\right)=0$ and $\Phi\left(x_{I}\right)=\Phi_{I}$. It is convenient to express $\rho_{\phi}\left(x=x_{I}\right)$ in terms of the expansion rate at $x_{I}$, which leads to

$$
\Phi_{I}=\frac{3}{8 \pi} \frac{M_{P l}^{2}}{M_{\phi}^{2}} \frac{H_{I}^{2}}{M_{\phi}^{2}} x_{I}^{3} .
$$

The numerical value of $x_{I}$ is irrelevant.

Before numerically solving the system of equations, it is useful to consider the early-time solution for $R$. Here, by early time, we mean $H \gg \Gamma_{\phi}$, i.e., before a significant fraction of the comoving coherent energy density is converted to radiation. At early times $\Phi \simeq \Phi_{I}$, and $R \simeq X \simeq 0$, so the equation for $R^{\prime}$ becomes $R^{\prime}=c_{1} x^{3 / 2} \Phi_{I}^{1 / 2}$. Thus, the early time solution for $R$ is simple to obtain

$$
R \simeq \frac{2}{5} c_{1}\left(x^{5 / 2}-x_{I}^{5 / 2}\right) \Phi_{I}^{1 / 2} \quad\left(H \gg \Gamma_{\phi}\right) .
$$

Now we may express $T$ in terms of $R$ to yield the early-time solution for $T$ :

$$
\begin{gathered}
\frac{T}{M_{\phi}} \simeq\left(\frac{12}{\pi^{2} g_{*}}\right)^{1 / 4} c_{1}^{1 / 4}\left(\frac{\Phi_{I}}{x_{I}^{3}}\right)^{1 / 8}\left[\left(\frac{x}{x_{I}}\right)^{-3 / 2}-\left(\frac{x}{x_{I}}\right)^{-4}\right]^{1 / 4} \\
\left(H \gg \Gamma_{\phi}\right) .
\end{gathered}
$$

Thus, $T$ has a maximum value of

$$
\begin{aligned}
\frac{T_{M A X}}{M_{\phi}} & =0.77\left(\frac{12}{\pi^{2} g_{*}}\right)^{1 / 4} c_{1}^{1 / 4}\left(\frac{\Phi_{I}}{x_{I}^{3}}\right)^{1 / 8} \\
& =0.77 \alpha_{\phi}^{1 / 4}\left(\frac{9}{2 \pi^{3} g_{*}}\right)^{1 / 4}\left(\frac{M_{P l}^{2} H_{I}}{M_{\phi}^{3}}\right)^{1 / 4},
\end{aligned}
$$

which is obtained at $x / x_{I}=(8 / 3)^{2 / 5}=1.48$. It is also possible to express $\alpha_{\phi}$ in terms of $T_{R H}$ and obtain

$$
\frac{T_{M A X}}{T_{R H}}=0.77\left(\frac{9}{5 \pi^{3} g_{*}}\right)^{1 / 8}\left(\frac{H_{I} M_{P l}}{T_{R H}^{2}}\right)^{1 / 4} .
$$

For an illustration, in the simplest model of chaotic inflation $H_{I} \sim M_{\phi}$ with $M_{\phi} \simeq 10^{13} \mathrm{GeV}$, which leads to $T_{M A X} / T_{R H} \sim 10^{3}\left(200 / g_{*}\right)^{1 / 8}$ for $T_{R H}=10^{9} \mathrm{GeV}$.

We can see from Eq. (11) that for $x / x_{I}>1$, in the earlytime regime $T$ scales as $a^{-3 / 8}$, which implies that entropy is created in the early-time regime [5]. So if one is producing a massive particle during reheating it is necessary to take into 
account the fact that the maximum temperature is greater than $T_{R H}$, and that during the early-time evolution, $T$ $\propto a^{-3 / 8}$.

\section{PRODUCTION OF A MASSIVE, STABLE PARTICLE SPECIES}

\section{A. Freeze out of the comoving $X$ energy density}

In this section we develop the equation for the $X_{F}$, the final value of $X$, which can be found from the early-time behavior.

At early times $\Phi \simeq \Phi_{I}$ and $R \simeq 0$. We will here also assume that $X \ll X_{E Q}$. Numerical results confirm the validity of this approximation and show that the massive particles are never in chemical equilibrium (although presumably they are in kinetic equilibrium). The early-time equation for the development of the $X$ energy density is

$$
X^{\prime}=c_{3} \Phi_{I}^{-1 / 2} x^{-5 / 2} X_{E Q}^{2} .
$$

$X_{E O}$ is given in terms of $M_{X}$ and the temperature, which may be found from the early-time solution for $R$.

We can integrate Eq. (15) by approximating it as a Gaussian integral. First we rearrange Eq. (15) by making appropriate redefinitions. Define the quantities $y$ and $\nu$ by $y \equiv X / x_{I}^{3}$ and $\nu \equiv\left(x / x_{I}\right)^{3 / 16}$. Now, using Eq. (12), we can rewrite Eq. (15) as

$$
y(\nu)=Q \int_{1}^{\nu} d \nu^{\prime} \exp \left[-H\left(\nu^{\prime}\right)\right]
$$

where we have defined

$$
\begin{aligned}
Q & \equiv \alpha_{\phi}^{3 / 4} \alpha_{X}\left(\frac{3^{1 / 2} 2^{1 / 4}}{\pi^{21 / 4} g_{*}^{3 / 4}}\right) \frac{M_{P l}^{3 / 2} M_{X}}{H_{I}^{1 / 4} M_{\phi}^{9 / 4}} \\
H(\nu) & \equiv \lambda\left(\nu^{-8}-\nu^{-64 / 3}\right)^{-1 / 4}-\frac{3}{4} \ln \left(\nu^{-8}-\nu^{-64 / 3}\right)-23 \ln \nu \\
\lambda & \equiv \frac{2^{5 / 4} \pi^{3 / 4} g_{*}^{1 / 4}}{3^{1 / 2}} \frac{\alpha_{\phi}^{-1 / 4} M_{X}}{M_{\phi}^{1 / 4} H_{I}^{1 / 4} M_{P l}^{1 / 2}} .
\end{aligned}
$$

To proceed with the Gaussian integral approximation, we assume $\nu_{0}^{-8} \gg \nu_{0}^{-64 / 3}$ where $\nu_{0}$ is the solution to $H^{\prime}\left(\nu_{0}\right)=0$. Then, we can easily solve $H^{\prime}\left(\nu_{0}\right)=0$, finding $\nu_{0}=\sqrt{17 / 2 \lambda}$, which is the point about which we Taylor expand $H(\nu)$ to quadratic order. Since the integrand falls to 0 rapidly away from $\nu=\nu_{0}$, and since we desire the freeze out value for $y$, the limits of the integrand can be taken to $\pm \infty$. We thereby find

$$
\frac{X_{F}}{x_{I}^{3}} \approx y_{\infty} \approx \frac{3^{5} \alpha_{\phi}^{3} \alpha_{X}}{8 \pi^{23 / 2} g_{*}^{3}}\left(\frac{H_{I}^{2} M_{P l}^{6}}{M_{X}^{8}}\right)\left(\frac{\sqrt{17}}{2}\right)^{17} \exp (-17 / 2) .
$$

Using Eq. (2), we rewrite this in a more transparent form

$$
\frac{X_{F}}{x_{I}^{3}} \approx \frac{4.21 \times 10^{-6}}{\left(g_{*} / 200\right)^{3 / 2}} \frac{\alpha_{X} H_{I}^{2} M_{P l}^{3} T_{R H}^{6}}{M_{\phi}^{3} M_{X}^{8}} .
$$

Note that this approximation should be valid as long as $\nu_{0}^{-8} \gg \nu_{0}^{-64 / 3}$ is satisfied. If the condition is not satisfied, the suppression will be exponential in $M_{X} / T$. Since one can express $\nu_{0}$ as

$$
\nu_{0} \approx \sqrt{\frac{17 T_{M A X}}{3 M_{X}}}
$$

the breakdown of the approximation simply indicates that the maximum temperature reached during reheating (which is much larger than the reheating temperature) has become much smaller than the mass of the $X$.

\section{B. $\Omega_{X} h^{2}$ in terms of $X_{F}$}

After freeze out of the comoving energy density of the stable particle, $X$ remains constant, so $\rho_{X}\left(x>x_{F}\right)$ $=X_{F} x_{I}^{-3} M_{X} M_{\phi}^{3}\left(x_{I} / x\right)^{3}$. For delayed reheating $\left(\Gamma_{\phi} \ll H_{I}\right)$ freeze out will be well before reheating. After reheating, $\rho_{X}\left(x>x_{R H}\right)=\rho_{X}\left(x_{R H}\right)\left(x_{R H} / x\right)^{3}$. The comoving entropy density is constant after reheating, so the radiation energy density scales as $\rho_{R}\left(x>x_{R H}\right)=\rho_{R}\left(x_{R H}\right)\left[g_{*}\left(T_{R H}\right) /\right.$ $\left.g_{*}(T)\right]^{1 / 3}\left(x_{R H} / x\right)^{4}$. Using these facts, we can express the present contribution of the massive particle species to the critical density in terms of the ratio of the energy densities at freeze out:

$$
\begin{aligned}
\frac{\Omega_{X} h^{2}}{\Omega_{R} h^{2}} & =\frac{\rho_{X}\left(T_{R H}\right)}{\rho_{R}\left(T_{R H}\right)}\left(\frac{g_{*}(\text { today })}{g_{*}\left(T_{R H}\right)}\right)^{1 / 3} \frac{x_{0}}{x_{R H}} \\
& =\frac{\rho_{X}\left(T_{R H}\right)}{\rho_{R}\left(T_{R H}\right)} \frac{T_{R H}}{T_{0}} \quad\left(\text { for } x>x_{R H}\right),
\end{aligned}
$$

where $x_{0}$ is the present value of $x$ and $T_{0}=2.37 \times 10^{-13} \mathrm{GeV}$ is the present temperature. ${ }^{2}$ Today, $\Omega_{R} h^{2}=4.3 \times 10^{-5}$, and the contribution to $\Omega h^{2}$ from the massive particle is

$$
\Omega_{X} h^{2}=1.5 \times 10^{18}\left(\frac{T_{R H}}{10^{9} \mathrm{GeV}}\right) \frac{X_{F}}{x_{I}^{3}} \frac{M_{X} M_{\phi}^{3}}{H_{I}^{2} M_{P l}^{2}} .
$$

Using the expression for $X_{F} / x_{I}^{3}$ from the previous section, we arrive at the final result

$$
\Omega_{X} h^{2}=M_{X}^{2}\langle\sigma|\mathrm{v}|\rangle\left(\frac{g_{*}}{200}\right)^{-3 / 2}\left(\frac{2000 T_{R H}}{M_{X}}\right)^{7} .
$$

\footnotetext{
${ }^{2}$ In this subsection we make the heretofore criticized approximation that the inflaton energy density scales like pressureless matter until it dumps all of its energy into radiation at the instant of "reheating." In this instance, however, it is an appropriate approximation, as borne out by analytic approximations and the numerical calculations presented in the next section.
} 


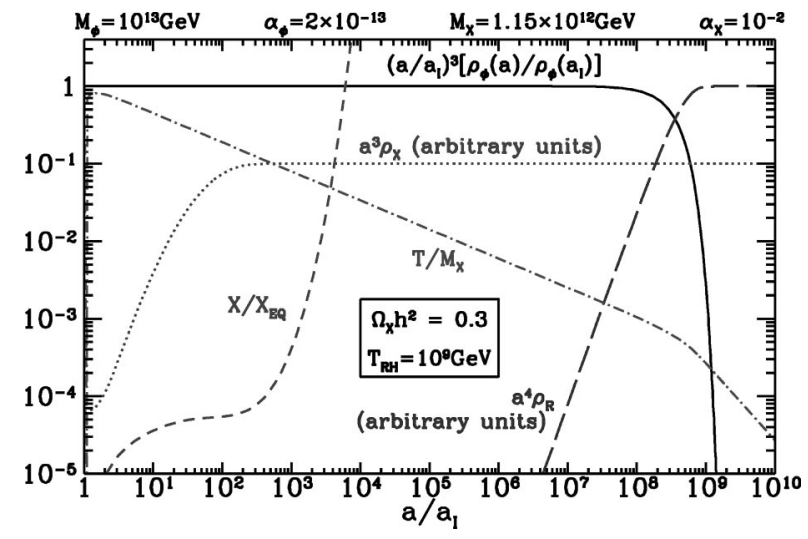

FIG. 1. The evolution of energy densities and $T / M_{X}$ as a function of the scale factor. Also shown is $X / X_{E Q}$.

\section{NUMERICAL RESULTS}

An example of a numerical evaluation of the complete system in Eq. (6) is shown in Fig. 1. The model parameters chosen were $M_{\phi}=10^{13} \mathrm{GeV}, \alpha_{\phi}=2 \times 10^{-13}, M_{X}=1.15$ $\times 10^{12} \mathrm{GeV}, \alpha_{X}=10^{-2}$, and $g_{*}=200$. The expansion rate at the beginning of the coherent oscillation period was chosen to be $H_{I}=M_{\phi}$. These parameters result in $T_{R H}=10^{9} \mathrm{GeV}$ and $\Omega_{X} h^{2}=0.3$.

We have made a particular choice for the reheat temperature for the numerical model illustrated in Fig. 1. The actual limit on the reheat temperature depends on the gravitino mass in a sensitive way. For instance, in the range $100 \mathrm{GeV}$ to $1 \mathrm{TeV}$ for the gravitino mass, the maximum reheat temperature ranges from $10^{7}$ to $10^{9} \mathrm{GeV}$, and for a gravitino mass in the range 1 to $3 \mathrm{TeV}$ it increases from $10^{9}$ to $10^{12}$ $\mathrm{GeV}$. Therefore, the value of the reheat temperature we used to illustrate the effect $\left(10^{9} \mathrm{GeV}\right)$ seems justified. The previous section contained analytical approximations expressed in term of the reheat temperature that can be applied for any value of $T_{R H}$.

Figure 1 serves to illustrate several aspects of the problem. Just as expected, the comoving energy density of $\phi$ (i.e., $a^{3} \rho_{\phi}$ ) remains roughly constant until $\Gamma_{\phi} \simeq H$, which for the chosen model parameters occurs around $a / a_{I} \simeq 5 \times 10^{8}$. But of course, that does not mean that the temperature is zero. Notice that the temperature peaks well before "reheating." The maximum temperature, $T_{M A X}=10^{12} \mathrm{GeV}$, is reached at $a / a_{I}$ slightly larger than unity (in fact at $a / a_{I}$ $=1.48$ as expected), while the reheat temperature, $T_{R H}$ $=10^{9} \mathrm{GeV}$, occurs much later, around $a / a_{I} \sim 10^{8}$. Note that $T_{M A X} \simeq 10^{3} T_{R H}$ in agreement with Eq. (14).

From the numerical results we can justify one of the assumptions in deriving the analytical approximations. From the figure it is clear that $X \ll X_{E Q}$ at the epoch of freeze out of the comoving $X$ number density, which occurs around $a / a_{I}$ $\simeq 10^{2}$. The rapid rise of the ratio after freeze out is simply a reflection of the fact that $X$ is constant while $X_{E Q}$ decreases exponentially. The relevance of the ratio is the justification of the neglect of $X_{E Q}$ term in Eq. (15).

Our numerical results also justify the assumption that the distribution function of the $X$-particles is well described by the non-relativistic function during the reheating process. In- deed, if the value of the momentum $p_{X}$ of the $X$-particles of the order of the temperature $T$, it is clear from Fig. 1 that $p_{X} / M_{X}$ is always smaller than unity. In particular we notice that when $T$ reaches its maximum, $p_{X} / M_{X} \simeq 0.8$. Had we chosen a higher value of $M_{X}$ while keeping $T_{R H}$ (and therefore $T_{M A X}$ ) fixed, this ratio would have been even smaller.

A close examination of the behavior of $T$ shows that after the sharp initial rise of the temperature, the temperature decreases as $a^{-3 / 8}$ [as follows from Eq. (12)] until $H \simeq \Gamma_{\phi}$, and thereafter $T \propto a^{-1}$ as expected for the radiation-dominated era.

For the choices of $M_{\phi}, \alpha_{\phi}, g_{*}$, and $\alpha_{X}$ used for the model illustrated in Fig. $1, \Omega_{X} h^{2}=0.3$ for $M_{X}=1.15 \times 10^{12}$ $\mathrm{GeV}$, in excellent agreement with the mass predicted by using Eq. (23).

\section{CONCLUSIONS}

Let us now analyze the implications of our findings for the grand unified theory (GUT) baryogenesis scenario, where the baryon asymmetry is produced by the baryon (or lepton) number violating decays of superheavy bosons [6]. At the end of inflation the universe does not contain any matter and, even more important, it is perfectly baryon symmetricthere is no dominance of matter over antimatter. This means that GUT baryogenesis may be operative only if the supermassive GUT bosons are regenerated during the stage of thermalization of the decay products of the inflaton field. ${ }^{3} \mathrm{~A}$ naive estimate would lead to conclude that that the maximum number density of a massive particle species $X$ produced after inflation is suppressed by a factor of $\left(M_{X} / T_{R H}\right)^{3 / 2} \exp \left(-M_{X} / T_{R H}\right)$ with respect to the photon number density. For such a reason, it is commonly believed that GUT baryogenesis is incompatible with models of inflation where the reheating temperature is much smaller than the GUT scale and, in general, than the mass of the $X$ particles, $T_{R H} \ll M_{X}$. In fact, we have seen that the reheat temperature has a limited meaning and should not be used as the maximum temperature obtained by the universe during reheating. The maximum temperature, Eq. (14), is much larger than $T_{R H}$, and particles of mass much greater than the eventual reheating temperature $T_{R H}$ may be created by the thermalized decay products of the inflaton without any exponential suppression factor. Indeed, the number density $n_{X}$ of particles $X$ after freeze out and reheating may be easily inferred from Eqs. (5) and (19), and reads

$$
\frac{n_{X}}{n_{\gamma}} \simeq 3 \times 10^{-4}\left(\frac{100}{g_{*}}\right)^{3 / 2}\left(\frac{T_{R H}}{M_{X}}\right)^{7}\left(\frac{M_{P l}}{M_{X}}\right) .
$$

In theories that invoke supersymmetry to preserve the flatness of the inflaton potential, the slow decay rate of the gravitinos, the superpartners of the gravitons, is a source of the cosmological problems because the decay products of the

\footnotetext{
${ }^{3}$ In the case in which reheating is anticipated by a period of preheating, superheavy bosons may be produced by the phenomenon of parametric resonance [7].
} 
gravitino will destroy the ${ }^{4} \mathrm{He}$ and $\mathrm{D}$ nuclei by photodissociation, and in the process destroy the successful nucleosynthesis predictions. The most stringent bound comes from the resulting overproduction of $\mathrm{D}+{ }^{3} \mathrm{He}$, which would require that the gravitino abundance is smaller than about $10^{-10}$ relative to the entropy density at the time of reheating after inflation. This translates into an upper bound on the reheating temperature after inflation, $T_{R H} / M_{P l} \lesssim 10^{-9}[4]$.

The calculation of the gravitino abundance is one example where the instantaneous reheat approximation works surprisingly well. We have checked both analytically and numerically that the quantity $\left(n_{3 / 2} / s\right) /\left(n_{3 / 2} / s\right)_{\text {final }}$ gradually increases with time when $\Gamma_{\phi}$ is smaller than $H$, but remains always smaller than unity until the inflaton decays at $t$ $\sim \Gamma_{\phi}^{-1}$. This means that most of the gravitinos are produced at the last stage of reheating when the inflaton decays and it is meaningful to talk about $T_{R H}$. We have also checked that to a very good approximation (and with a very weak dependence upon the inflaton mass) $\left(n_{3 / 2} / s\right)_{\text {final }}$ is equal to the usual estimate one obtains neglecting the nontrivial evolution of the temperature of the radiation during the period $\Gamma_{\phi}$ $\ll H$. This is because during the coherent oscillation epoch the entropy per comoving volume is increasing and the abundance of newly born gravitinos is continuously diluted by the entropy release.

Therefore, although a maximum temperature of $10^{12} \mathrm{GeV}$ seems to be in contradiction with the upper bound of $10^{9}$ to $10^{10} \mathrm{GeV}$ for $T_{R H}$, it should be clear that what really matters for the gravitino number density is the reheating temperature, and not the maximum temperature reached by the plasma. These considerations hold in the case in which the generated gravitinos are relativistic, $m_{3 / 2} \ll T_{R H}$. As the mass of the gravitinos is increased (or equivalently, as $T_{R H}$ is lowered) and they become non-relativistic, the gravitinos will be well described by the particles $X$, with the only difference that the parameter $\alpha_{X}$ will be suppressed by powers of the Planck scale.

It is easy to check that for small values of $T_{R H}$ the ratio in Eq. (24) is always much larger than the equilibrium value, $n_{X}^{E Q} / n_{\gamma}=\left(M_{X} / 2 T_{R H}\right)^{3 / 2}\left[\pi^{1 / 2} / \xi(3)\right] \exp \left(-M_{X} / T_{R H}\right)$. This result is crucial for the out-of-equilibrium decay scenarios of baryogenesis. For instance, in theories where $B-L$ is a spontaneously broken local symmetry, as suggested by $S O(10)$ unification, the cosmological baryon asymmetry can be generated by the out-of-equilibrium decay of the lightest heavy Majorana right-handed neutrino $N_{1}^{c}$ [8], whose typical mass is about $10^{10} \mathrm{GeV}$. For reheat temperatures of the order of $10^{9} \mathrm{GeV}$, the number density of the right-handed neutrino is about $3 \times 10^{-2} n_{\gamma}$ and one can estimate the final baryon number to be of the order of $B \sim\left(n_{N_{1}^{c}} / n_{\gamma}\right)\left(\epsilon / g_{*}\right) \simeq 10^{-4} \epsilon$, where $\epsilon$ is the coefficient containing one-loop suppression factor and $C P$ violating phases. The observed value of the baryon asymmetry, $B \sim 10^{-10}$, is then obtained without any fine tuning of parameters.

Our findings have also important implications for the conjecture that ultra-high cosmic rays above the GreisenZatsepin-Kuzmin cutoff of the cosmic ray spectrum, may be produced by decays of superheavy long-living particles
[9-11]. In order to produce cosmic rays of energies larger than about $10^{12} \mathrm{GeV}$, the mass of the $X$-particles must be very large, $M_{X} \gtrsim 10^{12} \mathrm{GeV}$, and their lifetime $\tau_{X}$ must be much larger than the age of the Universe, $\tau_{X} \geq 10^{10} \mathrm{yr}$. With the smallest value of the lifetime, the observed flux of ultrahigh energy cosmic rays will be reproduced with a rather low density of $X$-particles, $\Omega_{X} \sim 10^{-12}$. It has been suggested that $X$-particles can be produced in the right amount by usual collisions and decay processes taking place during the reheating stage after inflation if the reheat temperature never exceeded $M_{X}$ [10]. Again, this conclusion was reached assuming naively that that the maximum number density of a massive particle species $X$ produced after inflation is suppressed by a factor of $\left(M_{X} / T_{R H}\right)^{3 / 2} \exp \left(-M_{X} / T_{R H}\right)$ with respect to the photon number density. One then would conclude that the reheat temperature $T_{R H}$ should be in the range $10^{11}$ to $10^{15} \mathrm{GeV}$ [9]. This is a rather high value and leads to the gravitino problem in generic supersymmetric models. This is one reason alternative production mechanisms of these superheavy $X$-particles have been proposed [12-14]. However, our analysis shows that the situation is much more promising. Making use of Eq. (23), the right amount of $X$-particles to explain the observed ultra-high energy cosmic rays is produced for

$$
\left(\frac{T_{R H}}{10^{10} \mathrm{GeV}}\right) \simeq\left(\frac{g_{*}}{200}\right)^{3 / 14}\left(\frac{M_{X}}{10^{15} \mathrm{GeV}}\right),
$$

where we have assumed $\langle\sigma|\mathrm{v}|\rangle \sim M_{X}^{-2}$. Therefore, we conclude that particles as massive as $10^{15} \mathrm{GeV}$ may be generated during the reheating stage in abundances large enough to explain the ultra-high energy cosmic rays even if the reheat temperature satisfies the gravitino bound.

\section{ACKNOWLEDGMENTS}

D.J.H.C. and E.W.K. were supported by the DOE and NASA under Grant NAG5-7092.

\section{APPENDIX A: THERMALIZATION OF LIGHT DEGREES OF FREEDOM}

The form of the Boltzmann equations we use, e.g., Eq. (3), assumes that the light particle decay products of the inflaton field are in local thermodynamic equilibrium (LTE). In this appendix we discuss this assumption, and the implications if it is not valid.

Before discussing the validity of the assumption, it is useful to recall why the assumption was made. In the derivation of Eq. (3), one starts with an equation for the rate of change of the $X$ number density due to the process $\gamma \gamma \rightarrow X X$ with four-momentum conservation $p_{\gamma}+p_{\gamma}^{\prime}=p_{X}+p_{X}^{\prime}$ :

$$
\begin{aligned}
\dot{n}_{X}= & \int \frac{d^{3} p_{\gamma}}{2 E_{\gamma}} \int \frac{d^{3} p_{\gamma}^{\prime}}{2 E_{\gamma}^{\prime}} \int \frac{d^{3} p_{X}}{2 E_{X}} \int \frac{d^{3} p_{X}^{\prime}}{2 E_{X}^{\prime}}(2 \pi)^{-8} \delta^{4}\left(p_{\gamma}+p_{\gamma}^{\prime} b f\right. \\
& \left.-p_{X}-p_{X}^{\prime}\right) f_{\gamma}\left(p_{\gamma}\right) f_{\gamma}\left(p_{\gamma}^{\prime}\right)|\mathcal{M}|_{\gamma \gamma \rightarrow X X}^{2}+\cdots
\end{aligned}
$$


Here $f_{i}(p)$ is the phase-space density of particle species $i$ with momentum $p_{i}$, and $|\mathcal{M}|_{\gamma \gamma \rightarrow X X}^{2}$ is the square of the matrix element for the process $\gamma \gamma \rightarrow X X$. With the assumption that the light particles are in LTE, the product of the light particle phase-space densities is $f_{\gamma}\left(p_{\gamma}\right) f_{\gamma}\left(p_{\gamma}^{\prime}\right)$ $=\exp \left(-E_{\gamma} / T\right) \exp \left(-E_{\gamma}^{\prime} / T\right)$. This last product is, of course, simply $f_{X}^{E Q}\left(p_{X}\right) f_{X}^{E Q}\left(p_{X}^{\prime}\right)$, which, after some rearrangement of Eq. (A1), leads to a term for the creation of $X$ 's proportional to $\left(n_{X}^{E Q}\right)^{2}$ :

$$
\dot{n}_{X}=\langle\sigma|\mathrm{v}|\rangle\left(n_{X}^{E Q}\right)^{2}+\cdots
$$

(for complete details, see [3]).

The factor $\left(n_{X}^{E Q}\right)^{2}$ in Eq. (A2) is present because not every light-particle collision has sufficient center-of-mass energy to create an $X$ pair. If LTE is established with temperature $T<M_{X}$, the factor $\exp \left(-2 M_{X} / T\right)$ in $\left(n_{X}^{E Q}\right)^{2}$ represents the fraction of the collisions with center-of-mass energy above threshold, i.e., with $\sqrt{s}>2 M_{X}$.

A simple indication of whether thermalization occurs on a timescale shorter than the timescale for $X$ production is the ratio of the cross section for the thermalization reactions to the cross section for $X$ production. If the ratio is larger than unity, then thermalization of the light degrees of freedom is a good assumption.

The process of $X$ production involves a "hard" process, and the cross section will be $\alpha_{X} / M_{X}^{2}$, where $\alpha_{X}$ was defined in Eq. (4). In order to produce an equilibrium distribution from the original decay distribution it is necessary to change the number of particles. Therefore, the relevant cross section is the one for processes like $\gamma \gamma \rightarrow \gamma \gamma \gamma{ }^{4}$ Although the thermalization reaction is higher order in perturbation theory, it is a "softer" process, and radiation of a soft photon has a large cross section.

Without knowing the details of the interactions of the decay products, it is impossible to say with certainty how complete thermalization will be. But if the inflaton decay products have usual gauge interactions, the thermalization cross section will be larger than the $X$ production cross section, and thermalization of the inflaton decay products is likely.

Now let us explore the consequences if LTE of the light degrees of freedom is not obtained. If the light particles are not in LTE, then the factor $n_{X}^{E Q}$ in Eq. (A2) could simply be replaced by the more general factor $n_{\gamma}\left(E>M_{X}\right) .{ }^{5}$ Now let us make the extreme assumption that the light degrees of freedom never interact before $X$ production, and that they have the original (redshifted) momentum with which they were created in inflaton decay. Assume this original momentum is $M_{\phi} / \eta$. If $M_{\phi} / \eta$ is greater than $M_{X}$, then the $X$ production rate actually will be larger than the equilibrium rate since $n_{\gamma}\left(E>M_{X}\right)>n_{X}^{E Q}$, while if $M_{\phi} / \eta$ is less than $M_{X}$, then the $X$ production rate will be zero. The most reasonable assumption is that even if there is a large multiplicity in $\phi$ decay, a fair fraction of $M_{\phi}$ is carried by a few leading particles. So the effective value of $\eta$ is probably not too large.

The above analysis leads us to the conclusion that thermalization of the light degrees of freedom is likely unless the inflaton decay products themselves are very weakly coupled to everything. Even if thermalization does not occur, production of massive particles during reheating is not much different than our simple model suggests.

\footnotetext{
${ }^{4}$ Recall that $\gamma$ represents a light particle, not just a photon, so $\gamma$ may carry electric charge, color charge, etc.

${ }^{5}$ Of course in this case $\langle\sigma|\mathrm{v}|\rangle$ would not be a thermal average, but an average over the actual phase-space density.
}

[1] For a recent review, see D. H. Lyth and A. Riotto, Phys. Rep. 314, 1 (1999).

[2] For a review, see L. Kofman, hep-ph/9802285.

[3] E. W. Kolb and M. S. Turner, The Early Universe (AddisonWesley, Menlo Park, Ca., 1990).

[4] J. Ellis, J. Kim, and D. V. Nanopoulos, Phys. Lett. 145B, 181 (1984); L. M. Krauss, Nucl. Phys. B227, 556 (1983); M. Yu. Khlopov and A. D. Linde, Phys. Lett. 138B, 265 (1984).

[5] R. J. Scherrer and M. S. Turner, Phys. Rev. D 31, 681 (1985).

[6] E. W. Kolb and M. S. Turner, Annu. Rev. Nucl. Part. Sci. 33, 645 (1983); see also A. Riotto, lectures given at ICTP Summer School in High-Energy Physics and Cosmology, Trieste, Italy, 1998, hep-ph/9807454.

[7] E. W. Kolb, A. Linde, and A. Riotto, Phys. Rev. Lett. 77, 4290
(1996); E. W. Kolb, A. Riotto, and I. I. Tkachev, Phys. Lett. B 423, 348 (1998).

[8] M. Fukugita and T. Yanagida, Phys. Lett. B 174, 45 (1986).

[9] V. A. Kuzmin and V. A. Rubakov, Phys. At. Nucl. 61, 1028 (1998).

[10] V. Berezinsky, M. Kachelriess, and A. Vilenkin, Phys. Rev. Lett. 79, 4302 (1997).

[11] M. Birkel and S. Sarkar, Astropart. Phys. 9, 297 (1998).

[12] D. J. Chung, E. W. Kolb, and A. Riotto, Phys. Rev. D 59, 023 501 (1999).

[13] V. Kuzmin and I. I. Tkachev, Pis'ma Zh. Éksp. Teor. Fiz. 68, 259 (1998) [JETP Lett. 68, 271 (1998)].

[14] D. J. Chung, E. W. Kolb, and A. Riotto, Phys. Rev. Lett. 81, 4048 (1998). 